\section{$03-4.2$ \\ COGNITIVE RESERVE AND COGNITIVE DECLINE: ARE INDIVIDUAL SUB-COMPONENTS OF RESERVE DRIVING THE ASSOCIATIONS?}

doi:10.1136/jech.2011.142976a.98

${ }^{1} \mathrm{R}$ Marioni, ${ }^{* 2} \mathrm{~A}$ van den Hout, ${ }^{3} \mathrm{M}$ Valenzuela, ${ }^{1} \mathrm{C}$ Brayne, ${ }^{2} \mathrm{~F}$ Matthews, ${ }^{1}$ Function MRC Cognitive and Study Ageing. ${ }^{1}$ University of Cambridge, Cambridge, UK; ${ }^{2} \mathrm{MRC}$ Biostatistics Unit, Cambridge, UK; ${ }^{3}$ University of New South Wales, Sydney, Australia

Differences in potentially modifiable factors, such as education, occupation, and late-life social engagement have been associated with cognitive decline and dementia in late life. Whether there is a collective effect of these variables on cognition-cognitive reserve-is unclear. This talk will break reserve into its individual components and compare the importance of each factor in models of cognitive change.

Data came from 13004 participants of the Medical Research Council Cognitive Function and Ageing Study who were aged 65 years and over. Cognition was assessed at multiple waves over 16 years using the Mini-Mental State Examination. Cognitive change was assessed using a multi-state model that incorporated deaths, right censoring, state misclassification, and cognitive recovery. Cognition was treated as a discrete variable with three possible states: no impairment, slight impairment, and moderate to severe impairment.

Results suggest independent effects for education, occupation, and social engagement. A higher level of education and a more complex occupation were associated with a decreased risk of cognitive decline to a slightly impaired state but they accelerated the transition to death from a severely impaired cognitive state; increased social engagement protected from transitions to severe impairment.

These findings support the possibility that contributions to cognitive reserve or cognitive lifestyle can be made across the life-course.

\section{3-4.3 EDUCATION IS ASSOCIATED WITH THE DELAYED ONSET OF TERMINAL DECLINE}

doi:10.1136/jech.2011.142976a.99

${ }^{1} \mathrm{G}$ Muniz-Terrera, ${ }^{*} \mathrm{C}$ Brayne, ${ }^{1} \mathrm{~F}$ Matthews, ${ }^{2} \mathrm{CC} 75 \mathrm{C}$ Study Collaboration Group. ${ }^{1}$ MRC Biostatistics Unit, Cambridge, UK; ${ }^{2}$ University of Cambridge, Cambridge, UK

Factors such as education have been associated with both late-life cognitive ability and cognitive decline. How education relates to decline in the final stages of life is not clear. We examined the association between education and the onset of terminal decline in a population-based longitudinal study of ageing.

Participants came from the Cambridge City over 75 Cohort Study $(n=2166)$. They were at least 75 years old at baseline $(1985-1987)$ and registered at any of six selected primary care practices in the Cambridge City (UK) area. Participants were followed-up over a 21year period. Global cognitive function was assessed using the Mini Mental State Examination 2, 7, 9, 13, 17 and 21 years after baseline.
People with higher education had a slightly delayed onset of terminal decline. Cognitive performance 2 years before death and its rate of change before the onset of terminal decline varied across individuals. However, in the terminal phase, we did not find evidence of variability across individuals in rate of decline nor its change. Thus, more educated individuals experience a slightly shorter period of accelerated cognitive decline before death.

Two findings emerge from this cohort in which almost all individuals have died. The first is that education does appear to have a slight delay on the onset of terminal decline. The second is that once initiated, terminal decline is a relatively uniform process in those aged 75 and over. Such findings have implications in early life enhancement of cognitive function and planning for the end of life.

\section{3-4.4 HOW DOES EDUCATION AFFECT ASSOCIATIONS BETWEEN NEUROPATHOLOGY AND CLINICAL DEMENTIA?}

doi:10.1136/jech.2011.142976a.100

B Stephan, ${ }^{*}$ EClipSE Collaborative Members. University of Cambridge, Cambridge, UK

Almost everyone who dies in old age has some pathology in their brain at death, but does not necessarily die with dementia. Cognitive reserve factors such as education are thought to protect ("neuroprotection") or provide resilience ("compensation") against dementiarelated pathology. We investigated the neuroprotection and compensation hypotheses in a large population-based cohort study (Epidemiological Clinicopathological Studies in Europe; EClipSE).

EClipSE has harmonised longitudinal clinical data and neuropathology from three longstanding population-based studies that included post-mortem brain donation. These three studies started between 1985 and 1991. Number of years of education during earlier life was recorded at baseline in each study. Incident dementia was detected through follow-up interviews, complemented by retrospective informant interviews, death certificate data and linked health / social records (dependent on study) after death. Dementia-related neuropathologies were assessed in each study in a comparable manner based on the CERAD protocol. Eight hundred and seventy-two brain donors were included of whom $56 \%$ were demented at death.

Longer years in education were associated with decreased dementia risk and greater brain weight but had no relationship with the burden of neurodegenerative or vascular pathologies. The associations between neuropathological variables and clinical dementia differed according to the "dose" of education such that more education reduced dementia risk largely independently of severity of pathology.

In conclusion, more education in early life did not protect individuals from developing neurodegenerative and vascular neuropathology by the time they died but it did appear to mitigate the impact of pathology on the clinical expression of dementia before death. 